\title{
A NOTE ON CONJUGATE FUNCTIONS
}

\section{JOHN LAMPERTI ${ }^{1}$}

1. Statement of results. The purpose of this note is to discuss as simply as possible a generalization of the classical theorem of $M$. Riesz $^{2}$ on the integrability of the conjugate functions of functions in $L_{p}$, and to point out some of the consequences for Fourier series. Suppose that $\phi(t)$ is a non-negative, convex function defined for $t \geqq 0$ with $\phi(0)=0$ and $\phi(2 t) \leqq K \phi(t)$ for some constant $K$. Then the set of realvalued functions $f(x)$ such that

$$
I[f]=\int_{0}^{2 \pi} \phi(|f(x)|) d x<\infty
$$

forms a vector space, which can be normed in several ways to form a Banach space (Orlicz space). Under our assumptions on $\phi$, these norms give equivalent topologies, which are also equivalent to the topology obtained by using the functional $I[f]$ to define convergence [4]; any of these (equivalent) spaces will be denoted by $L_{\phi}$. We shall extend Riesz's theorem from $L_{p}$ spaces to a wide class of spaces $L_{\phi}$.

Let us define classes of functions $\phi(t)$ by supposing that in addition to the conditions mentioned above, $\phi$ also satisfies the following:

Class A: $\phi^{\prime}(t)$ is concave, and $\phi\left(t^{\theta}\right)$ is convex for some $\theta<1$.

Class B: $\phi^{\prime}(t)$ is convex, and $\phi\left(t^{1-\theta}\right)$ is concave for some $\theta<1$.

Class C: $\phi(t)=\phi_{1}(t)+\phi_{2}(t)$, where $\phi_{1} \in A$ and $\phi_{2} \in B$.

Class D: $0<a \leqq \phi(t) / \phi_{1}(t) \leqq b<\infty$ for all large $t$, where $\phi_{1} \in C$.

Class $\mathrm{E}: \phi(t)=t^{p} L(t)$ where $1<p \neq 2$ and $L(t)$ is slowly varying. ${ }^{3}$ Class $E$ is a class of functions of regular variation [1] and probably includes the most interesting special cases. It is obvious that $A \cup B \subset C \subset D$; we shall show later that also $E \subset D$.

THEOREM 1. Let $\phi(t)$ belong to any of the classes $A-E$. If $f(x) \in L_{\phi}$, so does the conjugate function $\bar{f}(x)$, and there is a constant $K$ such that

$$
\|\bar{f}(x)\|_{\phi} \leqq K\|f(x)\|_{\phi} .
$$

Received by the editors June 4, 1958 and, in revised form, July 16, 1958.

1 I wish to acknowledge my indebtedness to $\mathrm{K}$. de Leeuw for valuable discussions.

2 See [5, Chapter vii]; for convenience, references will be made to [5] throughout, rather than to the original papers.

'In this paper, "slowly varying" will be interpreted in Karamata's sense to mean that $L(c t) / L(t) \rightarrow 1$ as $t \rightarrow \infty$ for all $c>0$. 
Theorem 1 is related to other work in various ways. ${ }^{4}$ Two further theorems in [5] suggest a method and give some results in this direction (see the remark following Lemma 2 and the proof of Lemma 3 below). Similar ideas are used by Lozinski [6] who obtains results containing those of Lemmas 1-3; his arguments yield considerably more than that but are also more involved. On the other hand, a different approach in the form of an interpolation theorem of Marcinkiewicz [7] (a proof and further results are given by Zygmund in [8]) yields a result [8, Theorem 2 and its corollary, Theorem 3] implying that (1) holds for a class of functions containing class E; however, the theorem employed is a rather deep one. It is believed that the present approach uses the simplest means to our specific ends.

Each of the following corollaries can be proved from the above theorem by essentially the same argument used in proving the $L_{p}$ version from Riesz's theorem; each has been known in a form employing Cesaro summability instead of convergence [5].

Corollary 1. If $\phi(t)$ satisfies the conditions of Theorem 1 , and $s_{n}(x)$ is the nth partial sum of the Fourier series of $f(x) \in L_{\phi}$, then

$$
\lim _{n \rightarrow \infty}\left\|f(x)-s_{n}(x)\right\|_{\phi}=0 .
$$

Corollary 2. Let $\phi(t)$ satisfy the conditions of Theorem 1, and let $\psi(t)$ be the Young complementary function. Let $a_{n}, b_{n}$ be the Fourier coefficients of a function $f(x) \in L_{\phi}$, and $a_{n}{ }^{\prime}, b_{n}{ }^{\prime}$ those of $g(x) \in L_{\psi}$. Then Parseval's formula

$$
\int_{0}^{2 \pi} f(x) g(x) d x=a_{0} a_{0}^{\prime} / 2+\sum_{n=1}^{\infty}\left(a_{n} a_{n}^{\prime}+b_{n} b_{n}^{\prime}\right)
$$

is valid with the series on the right convergent.

Corollary 3. Under the conditions of Corollary 2, the complex form of the Fourier series of $f(x) g(x)$ is the formal product of the complex Fourier series of $f(x)$ with that of $g(x)$, where the series defining the coefficients of the product are convergent.

An interpretation of Corollary 1 is that the trigonometric functions form a basis for the Banach space $L_{\phi}$. In the $L_{p}$ case, $1<p \neq 2, \mathrm{~S}$. Karlin [2] has shown that they do not form an absolute basis, and the corresponding result holds for some spaces $L_{\phi}$ :

4 The author is indebted to the referee for calling his attention to $[6 ; 7]$, and [8]. 
THEOREM 2. If $\phi(t)$ belongs to class $E$, the trigonometric functions $\{\sin n x, \cos n x\}$ form a nonabsolute basis for $L_{\phi}$.

Proof. As in [2] it is enough to consider $1<p<2$. Then there is a function $f(x)$ in $L_{\phi}$ but not in $L_{2} ; f(x)$ is also in $L_{p_{1}}$ if $1<p_{1}<p$. If the trigonometric functions were an absolute basis for $L_{\phi}$, the finite subseries of the Fourier series of $f(x)$ would be uniformly bounded in the $L_{\phi}$ norm, and hence also in the $L_{p_{1}}$ norm. But the proof of Karlin's theorem [2, p. 980] shows that then $f(x)$ would have to belong to $L_{2}$, a contradiction.

2. Proof of theorem 1. As in the case of Riesz's theorem, it is enough in view of Theorem 4.36 (iii) of [5] to prove that if $F(z)$ $=u(z)+i v(z)$ is analytic for $|z|<1$ and $v(0)=0$, then

$$
I\left[F\left(r e^{i x}\right)\right] \leqq K^{\prime} I\left[u\left(r e^{i x}\right)\right], \quad 0 \leqq r<1 .
$$

(Actually, it is easy to see that the operation mapping $f(x)$ into $\bar{f}(x)$ is closed, and so if $\bar{f}(x) \in L_{\phi}$ whenever $f(x)$ does, (1) follows from the closed graph theorem. We shall not need this observation, however, since (2) directly implies that the operation is continuous.) The basic idea of our proof is that Stein's proof [5, Chapter vii] of the classical theorem can be generalized. The proof will be carried out in a series of lemmas.

Lemma 1. If $\phi(t)$ belongs to Class A and $u(z)>0$ for $|z|<1$, then (2) holds with $K^{\prime}=(1-\theta)^{-1}$.

Proof. As in Stein's proof, we use Green's theorem

$$
\oint_{C} \frac{\partial w\left(r e^{i x}\right)}{\partial r} d s=\iint \Delta w d \sigma
$$

$s$

where $C$ is the circle $|z|=r$ and $S$ is its interior. Now

$$
\Delta \phi(u(z))=\phi^{\prime \prime}(u)\left|F^{\prime}(z)\right|^{2},
$$

and

$$
\Delta \phi(|F(z)|)=\left\{\phi^{\prime \prime}(|F|)+\phi^{\prime}(|F|) /|F|\right\}\left|F^{\prime}\right|^{2} .
$$

Since $\phi\left(t^{\theta}\right)$ is convex, $\phi^{\prime}(t) / t \leqq[\theta /(1-\theta)] \phi^{\prime \prime}(t)$, and also from the definition of Class $\mathrm{A} \phi^{\prime \prime}(t)$ is decreasing. Hence

$$
\Delta \phi(|F(z)|) \leqq \frac{1}{1-\theta} \Delta \phi(u(z)) .
$$

Applying (3) to $w=u\left(r e^{i x}\right)$ and $w=\left|F\left(r e^{i x}\right)\right|$ yields 


$$
r \frac{d}{d r} I\left[F\left(r e^{i x}\right)\right] \leqq \frac{1}{1-\theta} r \frac{d}{d r} I\left[u\left(r e^{i x}\right)\right], \quad r<1,
$$

and since $I[F(0)]=I[u(0)]$, (2) follows.

Lemma 2. If $\phi(t)$ belongs to Class $\mathrm{A}$, (2) holds with $K^{\prime}=2(1-\theta)^{-1}$.

Proof. The condition $\phi^{\prime}(t)$ concave implies that

$$
I[f+g] \leqq 2 I[f]+2 I[g] .
$$

With this in place of Minkowski's inequality, an argument like that in [5, p. 147] applies and the lemma follows.

REMARK. Theorem 7.6.6 of [5] actually contains the result we have proved so far except for formulating the definition of Class $\mathrm{A}$, and it could have been used to shorten the proof.

Lemma 3. Theorem 1 is true if $\phi(t)$ is in Class $\mathrm{B}$.

Proof. Let $\psi(t)$ be the Young complementary function of $\phi(t)$; it is not hard to see that if $\phi(t) \in B$, then $\psi(t) \in A$ and so by Lemma 2 the theorem holds for $L_{\psi}$. Theorem 7.6.5 of [5] therefore applies with $\Phi=\Phi_{1}=\psi$ and $\Psi=\Psi_{1}=\phi$ and yields the desired result.

\section{Lemma 4. Theorem 1 is true if $\phi(t)$ is in Class C.}

Proof. This is clear from the definition of Class $\mathrm{C}$ and what has already been shown.

Lemma 5. Theorem 1 is true if $\phi(t)$ is in Class D.

Proof. From the definition of Class $\mathrm{D}$, there is a function $\phi_{1}(t)$ in Class $\mathrm{C}$ such that the functionals $I[f]$ formed using $\phi(t)$ and $\phi_{1}(t)$ determine the same definition of convergence. Hence the Banach spaces $L_{\phi}$ and $L_{\phi_{1}}$ have equivalent norms, and since the theorem has been proved for $L_{\phi_{1}}$ it holds also for $L_{\phi}$.

Class D is actually the most general class for which we shall prove the theorem, but it is not a very natural set of functions. We conclude by showing that the more attractive Class $E$ is in fact a subclass of $D$.

Lemma 6. Class D contains Class E.

Proof. We shall show first that if $\phi(t)=t^{p} L(t)$ with $1<p<2$ and $L(t)$ slowly varying, there is a function in Class A which plays the role of $\phi_{1}(t)$ to show that $\phi$ belongs to $D$. Now $\phi^{\prime}(t)$ is monotone since $\phi(t)$ was assumed convex, and so Theorem 2 of [3] applies and gives

$$
\phi^{\prime}(t)=p t^{p-1} c(t) L(t), \text { where } \lim _{t \rightarrow \infty} c(t)=1 .
$$


Combining this with Karamata's representation theorem for slowly varying functions [1] yields

$$
\phi^{\prime}(t)=t^{p-1} d(t) \exp \int_{1}^{t} \frac{\xi(x)}{x} d x,
$$

where as $t \rightarrow \infty, d(t) \rightarrow d \neq 0$ and $\xi(t) \rightarrow 0$. It follows that

$$
\log \left\{\left[\phi^{\prime}(t) / d(t)\right]^{\prime}\right\}=\int_{1}^{t} \frac{p-2+\xi(x)}{x} d x+\log (p-1+\xi(t)) .
$$

Now let $m$ be a number such that

$$
|\xi(t)| \leqq \min \left(\frac{2-p}{2}, \frac{p-1}{2}\right) \quad \text { for all } t \geqq m,
$$

and introduce

$$
\eta(t)=\xi(t) \text { if } t \geqq m, \quad \eta(t)=\xi(m) \text { if } t \leqq m .
$$

Define

$$
h(t)=\int_{1}^{t} \frac{p-2+\eta(x)}{x} d x+\log (p-1)
$$

and let

$$
\phi_{1}(t)=\int_{0}^{t} \int_{0}^{x} e^{h(y)} d y d x .
$$

It is not hard to see that $\phi_{1}(t)$ does what it is supposed to. In fact, for some constant $k \neq 0$

$$
\phi_{1}^{\prime}(t) \sim k \phi^{\prime}(t)
$$

so that the same is true for $\phi_{1}(t)$ and $\phi(t)$. Also,

$$
\phi_{1}^{\prime \prime}(t)=e^{h(t)} \frac{p-2+\eta(t)}{t}<0,
$$

so $\phi_{1}^{\prime}(t)$ is concave. Similarly our choice of $\eta(t)$ guarantees that $\phi_{1}\left(t^{\theta}\right)$ is convex if $\theta \geqq 2 /(p+1)$.

If $\phi(t)=t^{p} L(t)$ with $p>2$, we can apply the above result to the Young complementary function of $\phi(t)$; alternatively, a very similar construction can be carried out directly. This completes the proof of the lemma and of the theorem.

\section{REFERENCES}

1. M. J. Karamata, Sur un mode de croissance régulière, Bull. Soc. Math. France vol. 61 (1933) pp. 55-62. 
2. S. Karlin, Bases in Banach spaces, Duke Math. J. vol. 15 (1948) pp. 971-985.

3. J. Lamperti, An occupation time theorem for a class of stochastic processes, Trans. Amer. Math. Soc. vol. 88 (1958) pp. 380-387.

4. W. A. J. Luxemburg and A. C. Zaanen, Conjugate spaces of Orlicz spaces, Indag. Math. vol. 18 (1956) pp. 217-228.

5. A. Zygmund, Trigonometrical series, Warsaw, 1935.

6. S. Lozinski, On convergence and summability of Fourier series and interpolation processes, Mat. Sb. vol. 14 (1944) pp. 175-263.

7. J. Marcinkiewicz, Sur l'interpolation d'opérations, C. R. Acad. Sci. U.S.A. vol. 208 (1939) pp. 1272-1273.

8. A. Zygmund, On a theorem of Marcinkiewicz concerning interpolation of operations, J. Math. Pures Appl. vol. 35 (1956) pp. 223-248.

STANFORD UNIVERSITY 